\title{
QUANTITY INTELLIGENT RECKONING FOR PACKAGED GRANARY GRAIN BASED ON IMAGE PROCESSING
}

\author{
Ying Lin ${ }^{1,2}$, Yong Liu ${ }^{1}$, Yueheng Sun ${ }^{2, *}$, Yanhong Sun ${ }^{1}$ \\ ${ }^{1}$ College of management, Chongqing Jiao Tong University, Chongqing, China,400074; \\ ${ }^{2}$ School of Computer Science and Technology, Tianjin University, Tianjin, China, 300072; \\ * Corresponding author, Address: School of Computer Science and Technology, Tianjin \\ University, Tianjin, China, 300072,Tel: +86-22-27401016,Email: yhs@tju.edu.cn
}

Abstract: This paper presents a quantity intelligent reckoning approach for packaged granary grain based on image processing. The actual scene video was taken as the analysis object, and the dual-threshold Canny operator and the morphology processing method are used to extract the object grain bags' characteristic outline-- the boundary of the counter-band of light. Then, a counting algorithm which integrates mode theory and variance analysis technology is presented for the quantity second-judgment. Experimental results show that by accurately extracting the characteristic outline and counting the number of the characteristic outline, the algorithm presents an effective method for grain quantity detection with high recognition precision and efficiency.

Keywords: quantity intelligent reckoning, packaged granary grain, image processing, counting algorithm

\section{INTRODUCTION}

From the nearly 20 years' practice of storage grain regulatory in China, the quantity of grain reserves supervision and auditing is still manual regulation. Because of the geographical dispersion and the lack of supervision of reserve granary, it is difficult for management departments to carry on the effective supervision and investigation, leading to the virtual, false subsidies, theft, and other widespread phenomena, bringing the huge

Please use the following format when citing this chapter:

Lin, Y., Liu, Y., Sun, Y. and Sun, Y., 2009, in IFIP International Federation for Information Processing, Volume 294, Computer and Computing Technologies in Agriculture II, Volume 2, eds. D. Li, Z. Chunjiang, (Boston: Springer), pp. 1115-1123. 
economic losses to the country, and what is more serious is that it affects the national macro-manipulation and price stability. So it is necessary to seek convenient and effective tools for reserve granary grain quantity reckoning ( Lin Ying et al., 2007). And the conveniences of digital images acquisition, transmission and identifiability cause the grain quantity examination tool which is based on image pattern recognition to become an important technological measure that solves the problems of long-range automatic monitoring and auditing (M.D. Kelly., 1973). In this paper a computerized intelligent recognition technology to achieve the precious quantity of grain reserves was adopted.

The key of video-based grain reserves automatic monitoring and auditing technology is based on the result of actual scenes video image recognition to calculate the real-time quantity of grain reserves. The grain pile is cubic, and each bag of grain's weight is fixed. If the number of grain bags were distinguished, the total quantity of grain reserves was able to be calculated. Therefore, the key of packaged granary grain quantity intelligent reckoning is various superficial grain bags recognition and quantity reckoning (Pavlidis, T., 1982). Based on the analysis of grain reserves characteristic, an effective and convenient method to recognize the object grain bag' characteristic outline -- counter-band of light boundary was proposed (Chen Xiaochun et al., 2006; Luan Xin et al., 1999). In this paper we introduced a smart method of video-based packaged granary grain quantity intelligent reckoning to extract the characteristic outline and count the number of the characteristic outline (Chen-Chau Chu et al., 1993).

\section{INTELLIGENT DETECTION METHOD}

The key of image recognition is whether the computer accurately understood the image information and effectively extracted the characteristics of region of interests (ROI). By comparing the effects of various local edge detection operators dealing with the actual scene images, the dual-threshold Canny operator as a local edge operator was chosen, and a counting algorithm based on statistical theory was involved (Kakarala, R. et al., 1992).

This method includes following four major steps:

1. Format conversion. Fully considered the complexity of the image information and the efficiency of image processing, the actual acquisition of the RGB images were converted into gray image.

2. Noise elimination. Regarding the influence of noise, the value filtering was used to carry on smooth processing to gray images and improved the quality of the gray images. 
3. Characteristic outline extraction. Based on the characteristic outline of the counter-band of light boundary category of rectangle, the dual-threshold Canny operator and the morphology processing method were effectively extracted ROI region.

4. Outline statistical counting. On the basis of determining ROI, a simple pixel statistic counting algorithm was presented, to some extent reduce the computational time and the complexity of the intelligent reckoning.

\subsection{Space conversion}

In practical application, the gray image processing is quite convenient, and the processing efficiency is high. According to images throughout the different gray level, the value of two-dimensional function $f(x, y)$ can be denoted as the gray value of the coordinates $(x, y)$ (Rosenfeld, A. et al., 1979). The monitor collects images for the red, green, blue (RGB) form, and the grain bags cross-section characteristic information is relatively simple, so first of all the gray conversion formula $f(x, y)=0.3 R+0.59 G+0.11 B$ was used to convert from the RGB space to gray space, and the result was shown in Fig.1 and Fig.2.

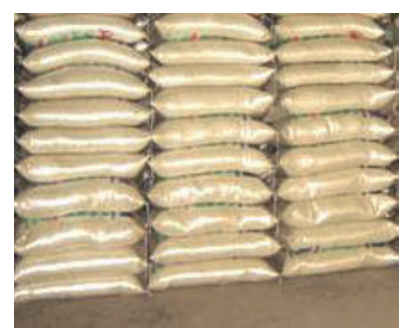

Fig.1: Original image

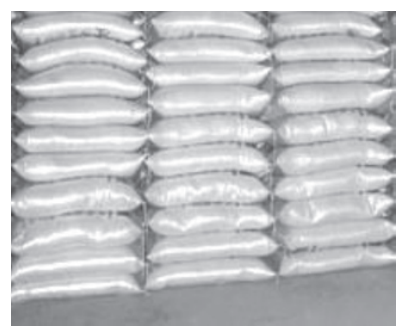

Fig 2: Gray image

\section{$2.2 \quad$ Image denoising}

The primitive packaged granary scene video images have a certain degree of noise pollution, making images become fuzzy, even blurred the image characteristic information. In order to improve the quality of the images, we often need to reduce or eliminate noise pollution for the accuracy of image recognition (Moulin, P. et al., 1999). Common methods of image enhancement, such as the neighbor average filtering, the median filtering, Wiener filtering, homomorphic filtering, and so on. Considered the complexity of the system software and the efficiency of image processing, the median filtering was adopted to carry on smooth processing with the scene gray images, and to eliminate noise pollution. By way of eliminating 
noise and preserving the information of characteristic outline effectively, a small $3 \times 3$ filter mask was used.

\subsection{Characteristic outline extraction}

The edge which is the partial characteristic discontinuous expression of the image depicts the characteristic outline. The main purpose of characteristic outline extraction is more accurately to identify the boundary of ROI in the scene image, on the basis of image pretreatment result acquired, in order to effectively improve the accuracy of characteristic outline extraction. According to the specialty of packaged-grain bags stacking in the reserve granary, the rectangular counter-band of light which was caused by the pressure among bags formed characteristic outline of each grain bag (John F. Haddon et al., 1990).

Characteristic outline extraction carried on the source gray image, using the rate of change of intensity and direction of changes in the suitable boundary segmentation local edge detection operator method checks each pixel point for the neighborhood, and the completion of the pixels in a neighborhood of gray rate of changes, which is in the direction of quantifying the identification, making the same rate of difference of gray pixels constitute closure and connectivity region. The main consideration of the fuzzy images of high level noise is how to choose the edge detection operator. For two-dimensional image $f(x, y), x, y$ respectively, on behalf of the pixels in a two-dimensional pixel-point benchmark of the abscissa, longitudinal coordinates, the position $f(x, y)$ of the gradient can be expressed as a vector, using $G_{x}$ and $G_{y}$. Specific formula as follows: Gradient vector can be expressed as the following

$$
\nabla \mathrm{f}=\left[G_{x}, G_{y}\right]^{T}=\left[\frac{\partial f}{\partial x} \frac{\partial f}{\partial y}\right]^{T}
$$

Set $\alpha(x, y)$ represent gradient direction

$$
\alpha(x, y)=\arctan \left(\frac{G_{x}}{G_{y}}\right)
$$

In the direction of $\alpha(x, y)$ the rate of change velocity will be

$$
\nabla f=\operatorname{mag}(\nabla \mathrm{f})=\left[G_{x}^{2}+G_{y}^{2}\right]^{\frac{1}{2}}
$$

In terms of practical application of the margin calculation, gradient operator is equivalent to the following calculated norm

$$
\nabla f=\left|G_{x}\right|+\left|G_{y}\right|=\left|\frac{\partial f}{\partial x}\right|+\left|\frac{\partial f}{\partial y}\right|
$$


Based on the characteristics of digital image processing and the analysis of actual scene images of the reserve granary, the form of a difference instead of the above calculation of the differential operator was used.

In the ideal circumstance, suppose the bending of each grain bag is the same, and the gathered counter-band of light image has the uniform width band of light. However, there are a lot of random factors in the actual packaged-grain bags stacking, such as the stacking-fault, changes in location of the bags, and so on, leading to the acquisition by showing a count-band of inhomogeneous width and local banding. Taking the complexity of the scene image into account, the first derivative may be unable to find the boundary and the information of the second derivative will be adopted. Five kinds of the edge detection algorithm were selected to analyze the scene image, and the results were shown in Fig.3. From the analysis of results, the Roberts algorithm was bad, lost many edges, and presented many isolated points. The Prewitt algorithm and the Sobel algorithm's effect were similar, and eliminated the noise well. But the local boundary presented the partial break. The LOG algorithm examined the characteristic outline of the counter-band of light well, but still presented the partial boundary break, and was relatively quite sensitive to the noise. The Canny algorithm obtained continual outline boundary, can distinguish characteristic of each grain bag's counter-band from the results, and effectively enhanced the anti-jamming capability (Marr, D. et al., 1980). In this paper we adopted the Canny edge detection operator to achieve the extraction of the characteristic outline and binaryzation.

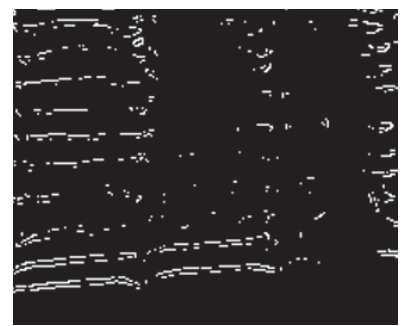

(a) Roberts algorithm

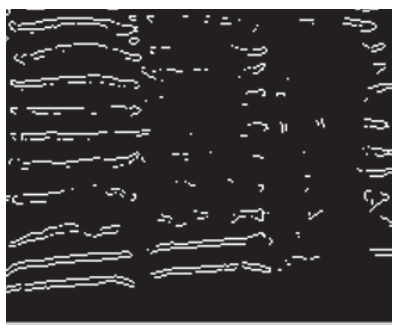

(b) Prewitt algorithm

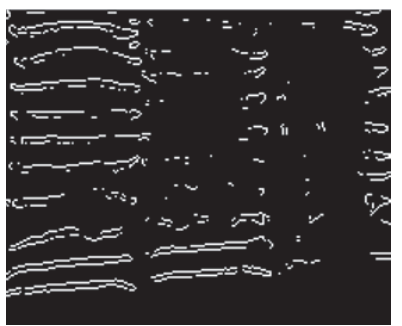

(c) Sobel algorithm

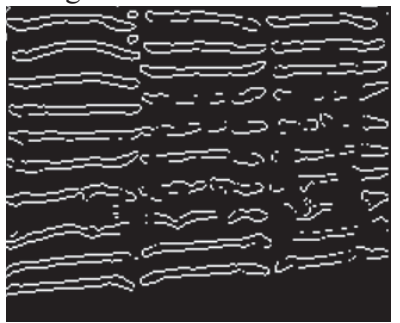

(d) LOG algorithm

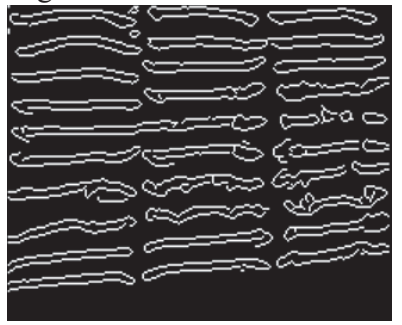

(e) Canny algorithm

Fig. 3: Comparison of counter-band of light boundary image edge detection algorithms 
In the actual process of reckoning, as the result of the limit of the scene light condition, the brightness and the contrast gradient of the images are non-uniform, and the boundary of the counter-band of light is not clear. There are two measures to improve the quality of the images: first, improve the scene illumination condition of the environment; second, take some effective measure to strengthen the ROI. In order to heighten the accuracy of reckoning, the full consideration in the design process is given. After the above image processing, we have already identified outline boundary of the counter-band of light of each grain bag, namely ROI region. But it still exist some noise spot which was misjudging as the pixels of ROI. The purpose of the outline enhancement is to wipe off the pixels of misjudgment and further identify the boundary of the counter-band of light. Based on morphological analysis of image processing, the specific structural element $B$ was introduced to refine $\mathrm{ROI}$ region $\mathrm{A}$ :

$$
A \otimes\{B\}=\left(\left(\cdots\left(\left(A \otimes B^{1}\right) \otimes B^{2}\right) \cdots\right) \otimes B^{n}\right)
$$

When the processing result of the region A no longer changed, the refinement finished. The result was shown in Fig.4.

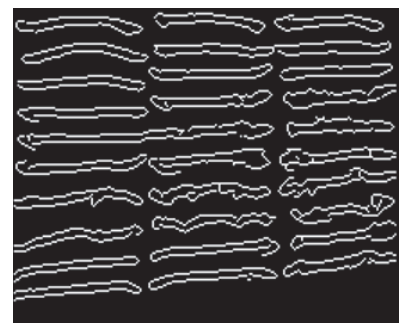

Fig 4: The extraction of characteristic outline boundary based on the Canny operator and the morphology processing method

\subsection{Outline statistical counting}

Pattern recognition of the identified ROI edge region was prepared for further grain quantity reckoning (Carlos et al., 1990). When the shape of characteristic outline was regular and disjunctive, and the boundary was clear, the corresponding template matching was used to identify its geometric parameters easily, such as central location, radius and so on ( Tang Jinkuan et al., 2007) . However, as the scene environment influence of some random factors and the outline of actual detection with some types of irregular rectangle, it is difficult to adopt the algorithm of template matching. Counting the number of each outline connected domain can effectively achieve the purpose with the exclusion of some complicated images which are sensitive to the noise. Fuzzy recognition method was restricted in the practical application because of the great computation load 
(Chen Songcan et al., 2000). Full consideration was given to the effectiveness of the recognition and the outline characteristic of the counterband of light, and we introduced a method based on a simple pixel statistic counting algorithm to achieve reckoning.

Assume $f(i, j)$ is the binary image of characteristic outline, whose size is $\mathrm{M} \times \mathrm{N}$, and $f(i, j)$ will range from 0 to 1 , where 0 denote the white pixels, and 1 the black pixels; $i \in\{0,1,2, \cdots, M-1\}, j \in\{0,1,2, \cdots, N-1\}$; Let [n] denote the maximal integer which is less than $n$ (Carlos et al., 1990). Check each pixel of the binary image followed by a vertical line from the top to bottom in turn, when the pixel value jump from 0 to 1 or 1 to 0 , accumulator plus one automatically. It is convenient to reckon the quantity of grain bags by accumulated value. Consider the timeliness of the algorithm, the measure of accumulation equipartition is adopted to select the position of vertical direction line segment, to avoid counting the vertical direction line segment of all. To improve the accuracy of reckoning, we selected many vertical directions to measure, introduced the analysis of combining the mode theory and the variance theory, set the corresponding threshold, and computed the final result. The algorithm is as follows:

1. Select vertical directions which will be

$$
x=\left[\frac{1}{n} M\right\rceil,\left\lceil\frac{2}{n} M\right],\left\lceil\frac{3}{n} M\right], \cdots,\left\lceil\frac{n-1}{n} M\right], n \geq 9
$$

check each pixel of the binary image followed by a vertical line from the top to bottom in turn, and write down various accumulation results, namely $k_{1}, k_{2}, k_{3}, \cdots, k_{n-1}$;

2. Count the number of the results of Step 1, let $k$ denote the mode of this group of data and $n_{0}$ the corresponding number, and we have frequency

$$
p=\frac{n_{0}}{n-1}
$$

3. If $p \geq 0.8$, then the variance will be

$$
\operatorname{Var}(k)=E\left\{\left[k_{i}-k\right]^{2}\right\}
$$

Where: $i=1,2, \cdots, n-1$, Or let $n=n+1$, and return to Step 1;

4. If $\operatorname{Var}(k)<T$, then stop computing and give the final result; Or let $n=n+1$, and return to Step 1, until the process finish. 


\section{THE EXPERIMENTAL ANALYSIS AND CONCLUSIONS}

In the actual quantity reckoning, according to the three neighboring surface images of the three-dimensional warehouse scene, the key technology to extracting various characteristic outlines and reckoning them was needed. Taking the timeliness and accuracy of the system into account, it is low efficiency to select so many initial vertical directions, whereas to select few directions will not achieve the high standard precision. Using the design methods in the actual recognition, all of the 20 samples of the characteristic outline can be correctly recognized, and the correct detection rate is $99 \%$. The experimental analysis indicates that the method used to achieve grain quantity intelligent reckoning is feasible.

From the above analysis, we can educe the conclusion of this intelligent reckoning, as follows:

1. Using the space conversion in pretreatment process, the image information can be suitably compressed, and the efficiency of intelligent reckoning is improved.

2. In view of the grain bags counter-band of light's characteristic boundary, using the concept of ROI, and integrating the dual-threshold Canny operator and the morphology processing method is adopted to achieve high-quality recognition.

3. It is the innovation which is based on a simple pixel statistic counting algorithm that is presented to meet management department's demand, and the advanced technology has certain value in practical application of the regulatory process of the reserve granary.

In view of the problems based on the reserve granary automatic video supervision and auditing, we presented a smart method that is through the pattern recognition of real-time scenes image to acquire the actual grain reserves. According to the characteristic of the counter-band of light, the dual-threshold Canny operator and the morphology processing method are integrated to achieve the extraction of ROI boundary. Then we presented a simple algorithm based on the pixel statistic counting to carry out the reliable quantity reckoning. However, as a result of the actual scene illumination limit and various grain bags characteristic's randomness, some boundary of processed images still has a certain shortage will influence highaccuracy of the quantity reckoning, therefore, the refinement of the characteristic outline boundary still need further study. 


\section{ACKNOWLEDGEMENTS}

This work is supported under the Bulk Warehouse Grain Quantity Recognition, a project funded by National Natural Science Foundation of China (Grant No.60603027) and Science \& Technology Commission of Chongqing Municipality, P.R. China (Grant No.2007 CE9006).

\section{REFERENCES}

Carlos A. Cabrelli and Ursula M. Molter. Automatic Representation of Binary Images, IEEE Transactions on Pattern Analysis and Machine Intelligence, 1990, 12(12):1190-1196

Chen Songcan, et al. Studies and Implementation of Fuzzy Recognition Methods for Image, ACTA ELECTRONICA SINICA, 2000, 28(11):50-54.

Chen Xiaochun, et al. A method of shape recognition, Pattern Recognition and Artificial Intelligence,2006,19(6):758-763. (in Chinese)

Chen-Chau Chu, Aggarwal, J.K. The integration of image segmentation maps using region and edge information. IEEE Transactions on Pattern Analysis and Machine Intelligence, 1993, 15(12):1241 - 1252

John F. Haddon, James F. Boyce. Image segmentation by unifying region and boundary information, IEEE Transactions on Pattern Analysis and Machine Intelligence, 1990, 12(10):929- 948 .

Kakarala, R., Hero, A.O. On achievable accuracy in edge localization, IEEE Transactions on Pattern Analysis and Machine Intelligence, 1992,14(7):777 - 781

Lin Ying, Fu Yang. The key of bulk warehouse grain quantity recognition ---- Rectangular benchmark image recognition, Journal of Zhejiang University(Engineering Science), 2007, 41(40): 1643-1646 (in Chinese)

Luan Xin, et al. The New Recognition Algorithm for Irregular Quasi circular Object. Journal of Image and Graphics, 1999, 4(3):202-206 (in Chinese)

M.D. Kelly, Edge Detection in Pictures by Computer Planning, Machine Intelligence, Vol. 6 (American Elsevier, New York, 1973), pp. 397-409.

Marr, D. \& Hildreth, E. Theory of Edge Detection, Proceedings of the Royal Society London, 1980, B207: 187-217

Moulin P, Liu J. Analysis of Multiresolution Image Denoising Schemes Using GeneralizedGaussian and Complexity Priors, IEEE Trans Information Theory, Special Issue on Multiscale Analysis, 1999, 45(3):909-919.

Pavlidis, T. (1982). Algorithms for Graphics and Image Processing, Computer Science Press, Maryland, USA.

Rosenfeld, A., Davis, L.S. Image segmentation and image models, Proceedings of the IEEE, 1979, 67(5):764 - 772

Tang Jinkuan, et al. A Novel Head-location Algorithm Based on Combined Mask, Journal of Image and Graphics, 2007, 12(8):1389-1394. (in Chinese) 